\title{
Possible role of EphA4 and VEGFR2 interactions in neural stem and progenitor cell differentiation
}

\author{
QINGFA CHEN $^{1 *}$, JIA LIU $^{2 *}$, TAKAHIRO SAWADA $^{3}$, CHUANFEI WEI $^{1}$, SHICHAO WU $^{1}$ and FABIN HAN ${ }^{1}$ \\ ${ }^{1}$ Centre for Tissue Engineering and Regenerative Medicine; ${ }^{2}$ Department of Neurology, Liaocheng People's Hospital, \\ Liaocheng University, Liaocheng, Shandong 252000, P.R. China; ${ }^{3}$ Department of Molecular Cell Biology and \\ Molecular Medicine, Institute of Advanced Medicine, Wakayama Medical University, Kimiidera, Wakayama 641-8509, Japan
}

Received January 25, 2019; Accepted December 4, 2019

DOI: $10.3892 /$ etm.2020.8419

\begin{abstract}
Neural stem and progenitor cells (NSPCs) are important pluripotent stem cells, which have potential applications for cell replacement therapy. Ephrin receptors (Ephs) and angiogenic growth factor receptors have a major impact on the proliferation and differentiation of NSPCs. Potential interactions between EphA4 and vascular endothelial growth factor (VEGF) receptor (VEGFR) 2, and their roles in NSPC differentiation in vitro remain unknown. In the present study, mouse embryonic NSPCs were treated with ephrin-A1 or VEGF165 alone as well as with combination treatment (ephrin-A1 + VEGF165). Immunoprecipitation and immunoblot assays demonstrated that wild-type EphA4, but not the EphA4 kinase-dead mutant, interacted with VEGFR2 when overexpressed in $293 \mathrm{~T}$ cells. This interaction was inhibited by dominant-negative EphA4. The percentage of $\beta$-tubulin III $(\text { Tuj1 })^{+}$, but not glial fibrillary acid protein $\left(\right.$GFAP) ${ }^{+}$cells, was increased in the ephrin-A1 + VEGF165 combination group as compared to the VEGF165 alone group in mouse embryonic NSPCs. VEGF165-induced neuronal differentiation was potentiated by ephrin-A1 in NSPCs in vitro and ephrin-A1- or VEGF165-stimulated EphA4 and VEGFR2 interactions may mediate the signaling pathway.
\end{abstract}

\section{Introduction}

Neural stem and progenitor cells (NSPCs) have self-renewal, proliferative and differentiation capabilities (1). NSPCs can differentiate into neurons, astrocytes or oligodendrocytes, all

Correspondence to: Dr Fabin Han, Centre for Tissue Engineering and Regenerative Medicine, Liaocheng People's Hospital, Liaocheng University, 67 Dongchang West Road, Liaocheng, Shandong 252000, P.R. China

E-mail: fhan2013@gmail.com

${ }^{*}$ Contributed equally

Key words: ephrin receptor A4, vascular endothelial growth factor receptor 2, neural stem cell, differentiation of which constitute the brain tissue (2). Therefore, it is possible that they have the potential to generate new neurons to compensate for loss in neurological diseases and central nervous system injuries, including Alzheimer's (AD) and Parkinson's disease (PD) in addition to spinal cord and traumatic brain injury $(3,4)$. Therefore, strategies to promote the neuronal differentiation of NSPCs are being investigated to allow for NSPC-based therapies worldwide $(3,4)$. Accumulating evidence has demonstrated that neurogenesis is linked to angiogenesis by numerous growth factors, including vascular endothelial growth factor (VEGF), ephrins and angiogenic factors $(5,6)$.

Ephrin receptors (Ephs), which constitute the largest receptor family within the receptor tyrosine kinase superfamily, regulate numerous important physiological and developmental processes (7-9). Both Ephs and ephrins are classified into type $A$ and B subclasses (7). Ephrin-As bind with EphAs, while ephrin-Bs bind with EphBs. However, EphB2 and EphA4 can bind to both ephrin-As and ephrin-Bs. Ligand interaction with the cell membrane is through glycosyl phosphatidylinositol linkage for ephrin-As, and through a short cytoplasmic and transmembrane domain for ephrin-Bs. EphA4-mediated forward signaling regulates neuroblast and astrocyte organization in a neurogenic niche (10). EphA4 may protect against neuronal loss and reverse cellular aging (5). EphA signaling commits NSPCs to differentiate down a neuronal lineage (11).

Angiogenic growth factors such as VEGFs, platelet-derived growth factors (PDGFs), and fibroblast growth factors (FGFs) play important roles in the proliferation and differentiation of NSPCs. Implantation of collagen glycosaminoglycan has been reported to promote angiogenesis accompanied by neurogenesis through VEGF, FGF2 and PDGF-BB upregulation (12). As a major angiogenic factor, VEGF promotes neurogenesis in NSPCs in vitro and in the adult brain (13). VEGF also has neurotrophic and neuroprotective effects (14). VEGF exerts its function through the VEGF receptor (VEGFR) 2, which mediates most neuron-specific effects (15). VEGF mediates positive neurogenic effects of an enriched environment on the rate of adult rodent de novo neurogenesis (16).

Previous research has shown that EphA4 and PDGF receptors (PDGFRs) form a heterodimer, trans-phosphorylating each other after stimulation with their ligands, and that their interaction promotes mouse embryonic neural precursor cell 
proliferation (17). It is therefore important to examine whether EphA4 and VEGFR2 form a heterocomplex and their role in NSPC differentiation. Due to their functions, understanding the interactions between EphA4, angiogenic growth factor receptors and the associated signaling pathways are critical in de novo neurogenesis and neuroregeneration in the human brain.

\section{Materials and methods}

Reagents. Recombinant human VEGF165 (cat no. 293-VE), recombinant human ephrinA1-fragment crystallizable region (Fc; cat no. 6417-A1), and recombinant human immunoglobulin $\mathrm{G}(\mathrm{IgG})-\mathrm{Fc}$ (cat no. MAB110) were used (all R\&D systems, Inc.). Clustered ephrin-A1-Fc was oligomerized according to the manufacturer's instructions via incubation with recombinant human $\mathrm{IgG}-\mathrm{Fc}$ for $>1 \mathrm{~h}$ at $4^{\circ} \mathrm{C}$, following previous protocols (18).

Mice and ethics statement. A total of three pregnant female C57BL/6 mice (weight range, 25-35 g; age, 8 weeks) were supplied by the Laboratory Animal Center of Shandong University (license no. SYXK-2019-0005; Shandong, China), housed at an ambient temperature of $22 \pm 2^{\circ} \mathrm{C}, 12$-h light/dark cycle and 40-45\% relative humidity. Animals were allowed free access to food and water. All animal experiments were performed in accordance with the guidelines of the Liaocheng People's Hospital (Shandong, China), and were approved by the Ethics Committee of Liaocheng People's Hospital (Shandong. China).

Cell culture. 293T cells (• Clontech Laboratories, Inc.) were cultured in DMEM (Gibco; Thermo Fisher Scientific, Inc.) supplemented with 10\% FBS (Gibco; Thermo Fisher Scientific, Inc.) and $1 \%$ penicillin/streptomycin (Gibco; Thermo Fisher Scientific, Inc.). Mouse embryonic NSPCs were cultured as previously described (19). Briefly, the NSPCs obtained from dissected hippocampus on embryonic day 14.5 were passaged as neurospheres in DMEM/F12 (Gibco; Thermo Fisher Scientific, Inc.) supplemented with B27 (Gibco; Thermo Fisher Scientific, Inc.), penicillin/streptomycin (Gibco; Thermo Fisher Scientific, Inc.), FGF2 (Gibco; Thermo Fisher Scientific, Inc.) and epidermal growth factor (EGF; Gibco; Thermo Fisher Scientific, Inc.) at $37^{\circ} \mathrm{C}$ with $5 \% \mathrm{CO}_{2}$ for up to three generations (P3). For experimentation with ligands, the P3 neurospheres were adherently cultured and NSPCs were starved in serum-free medium containing $0.5 \%$ (m/v) BSA (Sigma-Aldrich; Merck KGaA) for $5 \mathrm{~h}$ prior to ligand stimulation when a differentiation assay was performed without EGF and FGF2.

Reverse transcription (RT)-quantitative (q)PCR. Mouse embryonic NSPCs dissociated from P3 neurospheres were rinsed with PBS after 3-day culture. The cells were homogenized using TRI reagent ${ }^{\circledR}$ (cat no. T9424; Sigma-Aldrich; Merck $\mathrm{KGaA}$ ) and total RNA was extracted according to the manufacturer's protocol. RT and subsequent PCR or qPCR were performed using the conditions as previously reported $(20,21)$. The forward and reverse primer sequences for RT-PCR and qPCR are shown in Table I.
NSPC differentiation and immunofluorescence. NSPC differentiation was performed based on a previously published method with minor changes (21). Briefly, $2 \times 10^{5}$ cells were plated on four-well chamber slides and incubated at $37^{\circ} \mathrm{C}$ in $5 \% \mathrm{CO}_{2}$ with vehicle control (PBS), VEGF165 (20 ng/ml) and/or clustered ephrin-A1-Fc $(0.5 \mu \mathrm{g} / \mathrm{ml})$, dissolved in growth factor-free DMEM/F12 medium for 7 days. For immunofluorescence analysis, dissociated NSPCs or neurospheres were fixed with $4 \%$ paraformaldehyde at room temperature for $1 \mathrm{~h}$, and blocked in $1 \%$ normal goat serum (Sigma-Aldrich; Merck KGaA) at room temperature for $1 \mathrm{~h}$. The cells were then incubated with mouse anti- $\beta$-tubulin III (Tuj1) monoclonal antibody (cat. no. T8660; 1:1,000; Sigma-Aldrich; Merck KGaA), rabbit anti- glial fibrillary acid protein (GFAP) polyclonal antibody (cat. no. Z0334; 1:1,1000; DAKO; Agilent Technologies, Inc.), rabbit anti-CD11 antigen-like family member B (CD11b) (cat. no. NB110-89474; 1:500; Novus Biologicals, Ltd.), mouse anti-Nestin monoclonal antibody (cat. no. ab22035; 1:500; Abcam), mouse anti-proliferation marker protein Ki-67 (Ki67; cat. no. P6834; 1:500; Sigma-Aldrich; Merck KGaA) and rabbit anti-transcription factor SOX-2 (SOX2; cat. no. BS-0523R; 1:500; Bio-Connect B.V.) at $4^{\circ} \mathrm{C}$ overnight, followed by incubation with the secondary antibodies, goat anti-mouse AlexaFluor ${ }^{\circledR}$ 488-conjugated (cat. no. 115-545-146; 1:1,000; Jackson ImmunoResearch Laboratories, Inc.) and goat anti-rabbit cyanine Cy3-conjugated IgG (cat. no. 711-165-152; 1:1,000; Jackson ImmunoResearch Laboratories, Inc.) for $2 \mathrm{~h}$ at room temperature in the dark. Nuclei were stained with Hoechst 33258 (1:10,000; Santa Cruz Biotechnology, Inc.) at room temperature for $5 \mathrm{~min}$. Cells (four fields of view/well) were examined (magnification, x200) under a Nikon fluorescence microscope (Nikon Ti-E; Nikon Corporation) or a confocal microscope (Olympus Corporation).

Plasmid transfection. The plasmids encoding the EphA4 (deletion of juxtamembrane domain, $\Delta \mathrm{JM}$; kinase dead, KD) mutant containing the deletion of 591-602 amino acids + V635M mutation (KD) and the VEGFR2 (KD) mutant containing K868R mutation were constructed using the QuickChange Lightning Site-Directed Mutagenesis kit (Stratagene; Agilent Technologies, Inc.) as per the manufacturer's protocols. Wild type (WT) EphA4-Flag, VEGFR2-HA, VEGFR2 (KD)-HA, EphA4 (KD)-Flag, EphA4( $\Delta \mathrm{JM}$, $\mathrm{KD})$-Flag and EphA4( $\Delta \mathrm{JM}, \mathrm{KD})$-GFP eukaryotic expression vectors were constructed as previously described through subcloning into the pcDNA3.1 vector $(18,22)$. Plasmids, including $0.5 \mu \mathrm{g} / \mathrm{ml}$ EphA4-Flag together with increasing concentrations $(1.0,2.0$ and $3.0 \mu \mathrm{g} / \mathrm{ml})$ of VEGFR2-HA, $0.5 \mu \mathrm{g} / \mathrm{ml}$ EphA4-Flag together with increasing concentrations $(0,0.5$ and $2.5 \mu \mathrm{g} / \mathrm{ml})$ of VEGFR2-HA or $0.5 \mu \mathrm{g} / \mathrm{ml}$ EphA4-Flag, $1.5 \mu \mathrm{g} / \mathrm{ml} \mathrm{VEGFR2-HA} \mathrm{together} \mathrm{with} \mathrm{increasing}$ concentrations $(0,0.5$ and $1.5 \mu \mathrm{g} / \mathrm{ml})$ of EphA4 $(\Delta \mathrm{JM}$, KD)-GFP were transiently transfected into $293 \mathrm{~T}$ cells using PerFectin (Genlantis, Inc.). EphA4 ( $\Delta \mathrm{J} M, \mathrm{KD})$-Flag and VEGFR2 (KD)-HA mutant eukaryotic expression vectors were subcloned into a pMXs-IRES-GFP retroviral vector (obtained from Dr T. Kitamura, University of Tokyo, Tokyo, Japan) and subsequently incorporated into retroviral particules by co-transfection with pMXs-IRES-GFP retroviral plasmids $(2.1 \mu \mathrm{g} / \mathrm{ml}$ for EphA4 mutant and $2.7 \mu \mathrm{g} / \mathrm{ml}$ for VEGFR2 
Table I. Sequences for each pair of PCR primers

\begin{tabular}{ll}
\hline Primer & \multicolumn{1}{c}{ Sequence (5'-3') } \\
\hline PDGFR $\alpha-F$ & GACGCACGCCAGACTGTGTATAAG \\
PDGFR $\alpha-R$ & TGCACCTCCACCACGAACTCTC \\
PDGFR $\beta-F$ & TGGAGATTCGCAGGAGGTCACC \\
PDGFR $\beta-R$ & GGCTTGCTTCTCGCTACTTCTGG \\
VEGFR1-F & GCAGCACCTTGACCTTGGACAC \\
VEGFR1-R & GACGGTGGCTTCGCAGTTCAG \\
VEGFR2-F & TCAGACAACAACCATTGGCGAGAC \\
VEGFR2-R & GCAGTGCCGACGAGGATAATGAC \\
GAPDH-F & CAAGGAGTAAGAAACCCTGGACC \\
GAPDH-R & CGAGTTGGGATAGGGCCTCT \\
Tuj1-F & CCTTCATCGGGAACAGCACG \\
Tuj1-R & ACTCCTCCTCGTCGTCTTCGTA \\
GFAP-F & CCAAGATGAAACCAACCT \\
GFAP-R & CGCTGTGAGGTCTGGCTT \\
$\beta$-actin-F & AGATGTGGATCAGCAAGCAG \\
$\beta$-actin-R & GCGCAAGTTAGGTTTTGCA
\end{tabular}

GFAP, glial fibrillary acidic protein; PDGFR, platelet derived growth factor receptor; Tuj, $\beta$-tubulin III; VEGFR, vascular endothelial growth factor receptor; $\mathrm{F}$, forward; $\mathrm{R}$, reverse.

mutant) and $0.4 \mu \mathrm{g} / \mathrm{ml}$ pCAGVSV-G vector (American Type Culture Collection) into 293T cells using FuGENE6 ${ }^{\circledR}$ (Roche Diagnostics $\mathrm{GmbH}$ ). Retroviruses for both mutants were harvested for subsequent NSPC infection as previously described $(16,18)$.

Immunoprecipitation and immunoblotting. Immunoprecipitation and immunoblotting were performed as previously described (21). 293T cells were extracted using lysis A buffer, containing $50 \mathrm{mM} 4$-(2-hydroxyethyl)-1-piperazineethanesulfonic acid buffer, $5 \mathrm{mM}$ ethylene diamine tetraacetic acid, $1 \%$ Triton X-100, $50 \mathrm{mM}$ sodium chloride, protease inhibitors $(1 \mu \mathrm{M}$ pepstatin A, $1 \mathrm{mM}$ phenylmethylsulfonyl fluoride, $1 \mu \mathrm{M}$ leupeptin, and $1 \mu \mathrm{M}$ aprotinin) and phosphatase inhibitors $(50 \mathrm{mM}$ sodium fluoride, $10 \mathrm{mM}$ sodium pyrophosphate, and $1 \mathrm{mM}$ sodium orthovanadate) as previously described (21). Following immunoprecipitation with specific antibodies using protein A agarose, the pellets were washed three times. Then immunoblotting was performed with diluted antibodies for $2 \mathrm{~h}$ at room temperature using a standard procedure (18). The following primary antibodies were used: Mouse anti-hemagglutinin (HA; 1:3,000; cat no. 11583816001, Roche Diagnostics $\mathrm{GmbH})$, mouse anti-FLAG ${ }^{\circledR}$ M2 (1:3,000; cat no. F3165; Sigma-Aldrich; Merck KGaA), mouse anti-hemagglutinin (HA; 1:3,000, cat no. sc-7392; Santa Cruz Biotechnology, Inc.), rabbit anti-EphA4 (1:3,000; cat no. sc-921; Santa Cruz Biotechnology, Inc.), mouse anti-EphA4 (1:3,000; cat no. sc-365503; Santa Cruz Biotechnology, Inc.), mouse anti-VEGFR2 (1:1,000; cat no. sc-393163; Santa Cruz Biotechnology, Inc.), mouse anti-phosphotyrosine (pY; clone $4 \mathrm{G}^{\circledR}{ }^{\circledR} ; 1: 1,000$; cat. no. 05-321, EMD Millipore) and mouse
anti-GFP (1:3,000; cat no. sc-9996; Santa Cruz Biotechnology, Inc.). Subsequently, membranes were incubated with a goat anti-mouse $\mathrm{IgG}$ horseradish peroxidase-conjugated (1:5,000; sc-2005, Santa Cruz Biotechnology, Inc.) or goat anti-rabbit IgG (1:5,000; sc-2004, Santa Cruz Biotechnology, Inc.) secondary antibody at room temperature for $2 \mathrm{~h}$. For detecting VEGF165-mediated tyrosine phosphorylation of VEGFR2, 293T cells were first starved for $5 \mathrm{~h}$ before stimulation with VEGF165 $(20 \mathrm{ng} / \mathrm{ml})$ for 0 and $10 \mathrm{~min}$. The immunoprecipitation for VEGFR2 and immunoblotting for pY were performed as aforementioned. To confirm reproducibility, experiments were performed at least three times.

Statistical analysis. Data were analyzed using GraphPad Prism 6 software (GraphPad Software, Inc.) using two-way ANOVA followed by Tukey's post hoc test for multiple comparisons. Data are displayed as the mean \pm SD. $\mathrm{P}<0.05$ was considered to indicate a statistically significant difference.

\section{Results}

Interactions and transphosphorylation between EphA4 and VEGFR2 in a kinase-dependent manner. Transient transfection of expression vectors for WT VEGFR2 and the expression vector for WT EphA4 or kinase-dead (KD) EphA4, a kinase-inactivated mutant form of EphA4 in which a Met residue was substituted for Val-653, was performed in 293T cells (Fig. 1A and B). VEGFR2 was found to interact with WT EphA4 but not with KD EphA4 from immunoblotting with anti-VEGFR2 antibodies followed by immunoprecipitation with anti-EphA4 antibodies. Phosphotyrosine analysis showed enhanced phosphorylation of WT EphA4 but not KD EphA4 with increasing doses of VEGFR2 (Fig. 1A and B). The results also demonstrated the interaction between EphA4 and VEGFR2, and the transphosphorylation in a protein dose-dependent manner (Fig. 1A). Based on these results, it was speculated that EphA4 interacts with VEGFR2 in a doseand kinase-dependent manner.

Inhibition of the interaction between EphA4 and VEGFR2 by an EphA4 dominant-negative mutant, EphA4 ( $\triangle J M, K D)$. EphA4 missing the juxtamembrane domain can bind to FGFR but loses kinase activity (18). To further confirm the interaction between EphA4 and VEGFR2, whether EphA4 $(\Delta \mathrm{JM}$, KD), a dominant negative mutant of EphA4, could inhibit binding of EphA4 to VEGFR2 was examined. Fixed amounts of VEGFR2 $(1.5 \mu \mathrm{g} / \mathrm{ml}$ per $6-\mathrm{cm}$ plate in $2 \mathrm{ml}$ culture medium) and WT EphA4 $(0.5 \mu \mathrm{g} / \mathrm{ml}$ per $6-\mathrm{cm}$ plate in $2 \mathrm{ml}$ culture medium) were co-expressed with incremental concentrations of EphA4 ( $\triangle \mathrm{JM}, \mathrm{KD})$ in 293T cells, and the binding of EphA4 to VEGFR2 was investigated. It was found that EphA4 ( $\Delta \mathrm{JM}$, KD) inhibited the interaction between EphA4 and VEGFR2 in a dose-dependent manner (Fig. 2A).

To further analyze whether there was a dominant-negative effect, the expression vector for EphA4 ( $\triangle \mathrm{JM}, \mathrm{KD})$ was transfected into 293T cells together with the expression vectors for EphA4 and VEGFR2. When fixed amounts of WT EphA4 and WT VEGFR2 were co-transfected in 293T cells, as shown in Fig. 2B, EphA4 ( $\triangle J M, K D)$ significantly suppressed VEGF165 mediated tyrosine phosphorylation of WT VEGFR2 both at 
A

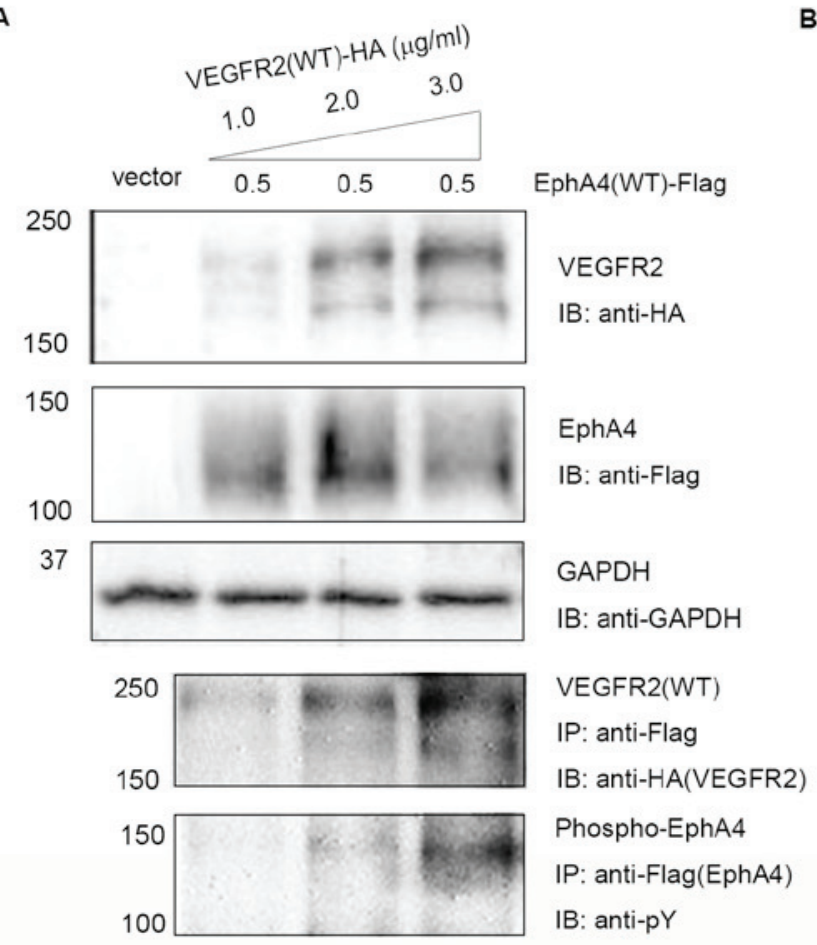

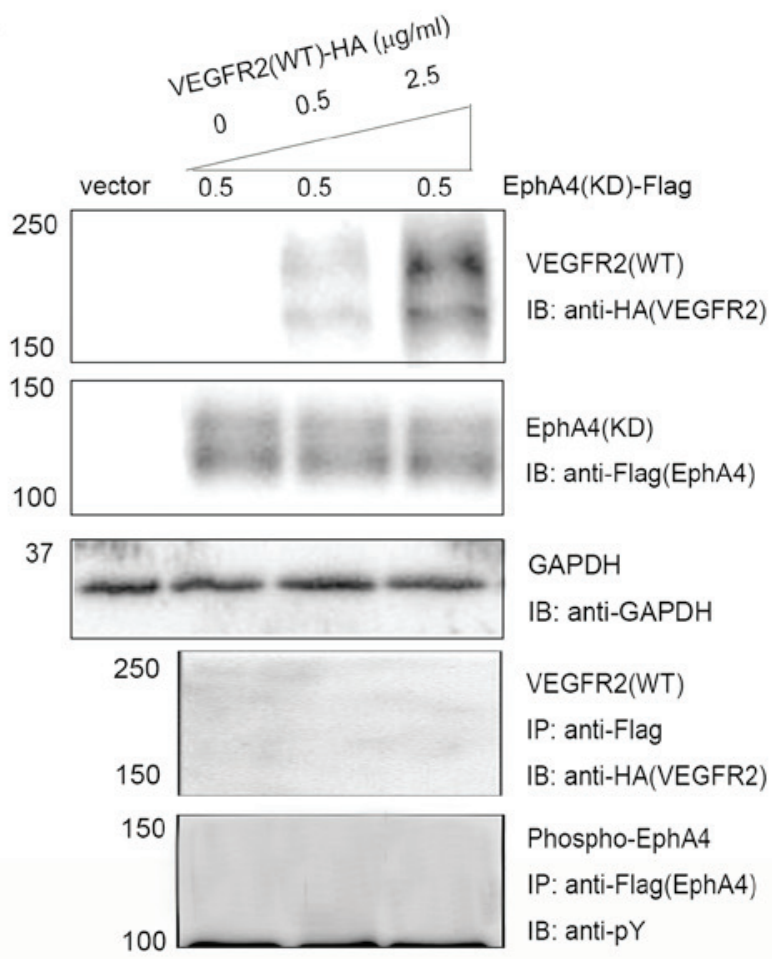

Figure 1. Complex formation and transphosphorylation of EphA4 and VEGFR2 in transfected 293T cells. (A) 293T cells were co-transfected with pcDNA/EphA4-Flag $(0.5 \mu \mathrm{g} / \mathrm{ml})$ and increasing concentrations $(1.0,2.0$ and $3.0 \mu \mathrm{g} / \mathrm{ml})$ pcDNA/VEGFR2-HA. (B) 293T cells were co-transfected with pcDNA/EphA4(KD)-Flag $(0.5 \mu \mathrm{g} / \mathrm{ml})$ and increasing concentrations $(0,0.5$ and $2.5 \mu \mathrm{g} / \mathrm{ml})$ of pcDNA/VEGFR2(WT)-HA. Interactions were detected using SDS-PAGE and IB using anti-Flag antibodies following with IP using anti-HA antibodies. Tyrosine phosphorylation of EphA4 was detected using immunoprecipitation with anti-Flag antibodies followed by immunoblotting with anti-pY anitbodies. Eph, ephrin receptor; HA, hemagglutinin; IB, immunoblotting; IP, immunoprecipitation; KD, kinase-dead; pY, phosphotyrosine; VEGFR, vascular endothelial growth factor receptor; WT, wild-type.

0 and 10 min (the peak of ligand stimulation). The results showed that the binding of EphA4 to VEGFR2 is important for both the EphA4 and VEGFR2 signaling pathways.

NSPC differentiation under ephrin-A1 and/or VEGF165 stimulation. To analyze the functional role of the interactions between EphA4 and VEGFR2, hippocampal cells from embryonic day 14.5 mice were cultured in DMEM/F12 serum-free medium supplemented with B27, FGF2 and EGF. Following one week of culture, the cells aggregated and formed spheroid neurospheres (Fig. 3A). Immunofluorescence staining revealed that cells within the neurospheres were immunoreactive to markers of neural stem cells nestin, Ki67 and SOX2 (Fig. 3B). Cells derived from the neurospheres were also immunoreactive to Tuj1, which is an immature neuronal marker, GFAP, an astrocyte marker and CD11b, an oligodendrocyte marker $(1,23)$ (Fig. 3C). These observations suggested that neurospheres derived from the hippocampus of embryonic mice exhibited active proliferative, self-renewal and multipotent properties in vitro.

The expression patterns of Ephs, VEGFRs and PDGFRs were investigated in the mouse embryonic NSPCs using RT-PCR. VEGFR family members (VEGFR1 and VEGFR2) and PDGFR family members (PDGFR $\alpha$ and PDGFR $\beta$ ) were detected in NSPCs (Fig. 3D).

Subsequently, VEGF165 (20 ng/ml) and/or clustered ephrin-A1-Fc $(0.5 \mu \mathrm{g} / \mathrm{ml})$ were added to the NSPC culture medium, and neuronal differentiation of NSPCs was evaluated using Tuj1 immunofluorescence (Fig. 3E). When compared with cells that were treated with PBS, the proportion of Tuj1 ${ }^{+}$cells increased significantly following stimulation with VEGF165 $(\mathrm{P}<0.05)$ or clustered ephrin-A1-Fc $(\mathrm{P}<0.05)$. The percentage of Tuj $1^{+}$cells exhibited a further increase under stimulation with clustered ephrin-A1-Fc + VEGF165 compared to cells with no stimulation $(\mathrm{P}<0.05)$ or stimulation with VEGF165 alone $(\mathrm{P}<0.05)$, suggesting enhanced neuronal differentiation of NSPCs when the cells were induced by simultaneous stimulation with the two ligands (Fig. 3E and F). Furthermore, the expression of Tuj1 in the vehicle-treated cells in the presence of VEGF165 and/or clustered ephrin-A1-Fc was markedly decreased in NSPCs after expression of a dominant-negative EphA4 mutant or expression of a kinase-negative VEGFR2 mutant. These data confirmed that ephrin-A1 promoted VEGF165-mediated neuronal differentiation of NSPCs (Fig. 3F).

The qPCR analysis indicated that the mRNA levels of Tuj1 were markedly upregulated following stimulation with VEGF165 $(\mathrm{P}<0.05)$, clustered ephrin-A1-Fc $(\mathrm{P}<0.05)$ and VEGF165 + clustered ephrin-A1-Fc $(\mathrm{P}<0.05)$ as compared to that of unstimulated control cells. The increased Tuj1 mRNA levels in the presence of VEGF165 and/or clustered ephrin-A1-Fc in the control cells were markedly inhibited with transfection of a dominant-negative EphA4 mutant or expression of a kinase-negative VEGFR2 mutant (Fig. 3G).

Glial differentiation from NSPCs was also analyzed using GFAP immunofluorescence (Fig. 3H). When compared with 
A
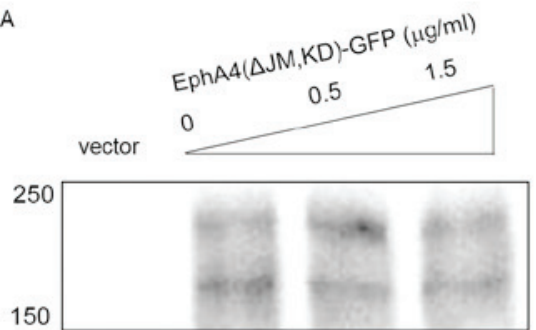

VEGFR2

IB: anti-HA

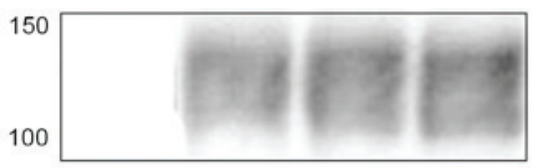

EphA4

IB: anti-Flag

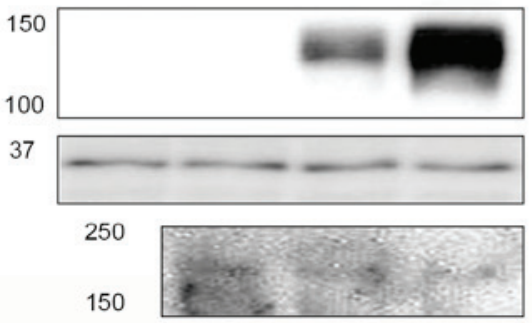

EphA4( $\triangle J M, K D)$-GFP

IB: anti-GFP

GAPDH

IB: anti-GAPDH

Binding

IP: anti-Flag

IB: anti-HA (VEGFR2)
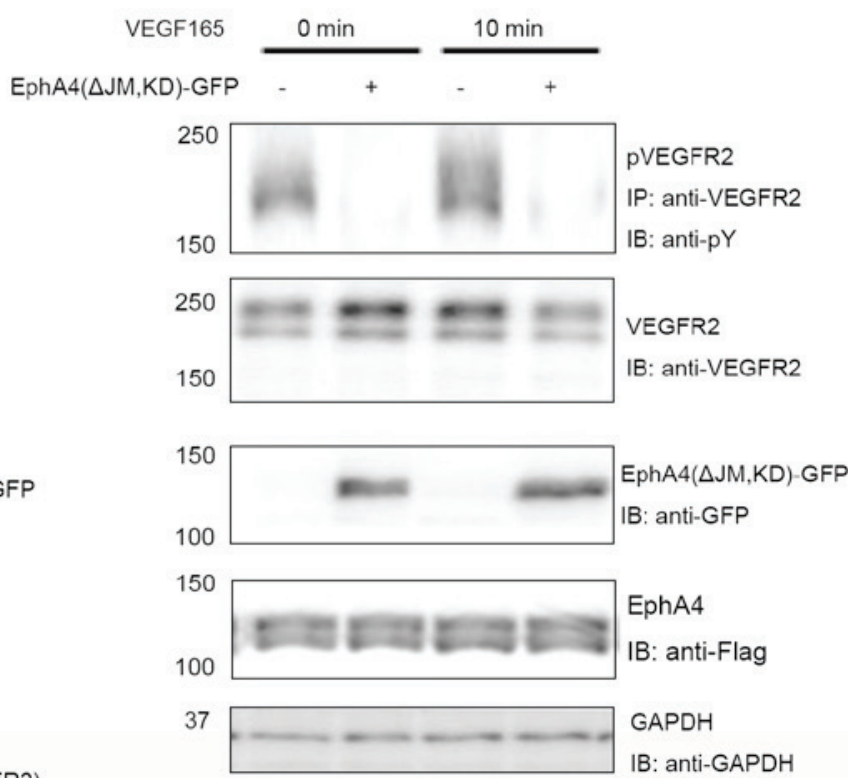

Figure 2. Inhibition of EphA4-VEGFR2 binding by EphA4( $\triangle \mathrm{JM}, \mathrm{KD})$-GFP. (A) EphA4-Flag and VEGFR2-HA were co-expressed with increasing doses of EphA4( $\triangle \mathrm{JM}, \mathrm{KD})$-GFP in 293T cells, and the binding of EphA4-Flag and VEGFR2-HA was examined using IB with or without IP using the antibodies shown following SDS-PAGE. (B) Inhibition of ligand-mediated receptor phosphorylation by EphA4( $\triangle \mathrm{JM}, \mathrm{KD})$ tagged with GFP. EphA4-Flag and VEGFR2-HA were co-expressed in 293T cells with or without EphA4( $\mathrm{JM}, \mathrm{KD})$-GFP, the phosphorylation of VEGFR2 was examined using IP with anti-VEGFR2 followed by IB with anti-pY following 0 or 10 min stimulation with $20 \mathrm{ng} / \mathrm{ml}$ VEGF165. $\triangle \mathrm{JM}$, juxtamembrane domain deleted; Eph, ephrin receptor; GFP, green fluorescent protein; HA, hemagglutinin; IB, immunoblotting; IP, immunoprecipitation; KD, kinase-dead; pY, phosphotyrosine; VEGF, vascular endothelial growth factor; VEGFR, VEGF receptor; WT, wild-type.

no stimulation, the proportion of $\mathrm{GFAP}^{+}$cells increased significantly following stimulation with VEGF165 $(\mathrm{P}<0.05)$ but not with clustered ephrin-A1-Fc stimulation. The percentage of $\mathrm{GFAP}^{+}$cells exhibited a slight but not significant increase under stimulation with clustered ephrin-A1-Fc + VEGF165 as compared to stimulation with VEGF165 alone (Fig. 3H and I). Furthermore, the expression of GFAP was markedly decreased in NSPCs after expression of a dominant-negative EphA4 mutant or expression of a kinase-dead VEGFR2 mutant. These results confirmed that ephrin-A1 promoted VEGF165-mediated glial differentiation of NSPCs (Fig. 3I).

The qPCR analysis indicated that the mRNA levels of GFAP were significantly upregulated following stimulation with VEGF165 $(\mathrm{P}<0.05)$ and VEGF165 + clustered ephrin-A1-Fc $(\mathrm{P}<0.05)$ as compared to that of unstimulated control cells. The increased GFAP mRNA levels in the presence of VEGF165 and/or clustered ephrin-A1-Fc were markedly reduced when cells were transfected with a dominant-negative EphA4 mutant or a kinase-dead VEGFR2 mutant (Fig. 3J). These results are consistent with the findings aforementioned in non-neuronal cells using ectopically expressed molecules (Figs. 1 and 2).

\section{Discussion}

The present study showed that EphA4 and VEGFR2 bind together in a dose and kinase-dependent manner when transiently co-expressed in the same cells. A dominant-negative molecule of EphA4 can inhibit the interaction between EphA4 and VEGFR2. Ephrin-A1 alone only produced a minor effect on NSPC differentiation. However, when ephrin-A1 and VEGF165 were added together, ephrin-A1 could potentiate VEGF-165-induced NSPC differentiation, revealing that ephrin-A1-stimulated EphA4 and VEGFR2 interactions may mediate the signaling pathway.

Previous research showed that EphA4 and PDGFRs or EphA4 and FGFRs could form a heterodimer, trans-phosphorylating each other when overexpressed in 293T cells or after stimulation with their ligands $(17,18)$. In the present study, a kinase-dependent interaction between EphA4 and VEGFR2 was found. Specifically, no interaction and trans-activation between the two molecules was detected when their kinase-dead mutants were overexpressed in $293 \mathrm{~T}$ cells. In addition to the Ephs measured in the NSPCs in a previous report (21), VEGFRs (VEGFR1 and VEGFR2) and PDGFRs (PDGFR $\alpha$ and PDGFR $\beta$ ) were also found to be expressed in the NSPCs. It was also found that NSPCs derived from the embryonic mouse brain respond to ephrin-A1 and VEGF165, promoting differentiation, and the effect of these two ligands was inhibited by the expression of a dominant-negative mutant of EphA4. The kinase-dead EphA4 abrogates the effect of VEGF165 and/or ephrin on NSPC differentiation by constitutively binding to VEGFR2. VEGF and ephrin-A1 enhanced the multipotent differentiation ability of NSPCs into neurons and astrocytes; however, limited detection of oligodendrocyte differentiation of NSPCs was observed using the routine differentiation protocol due to the absence of additional essential supplements that are required for oligodendrocyte differentiation (24). Together with the finding that the EphA4 


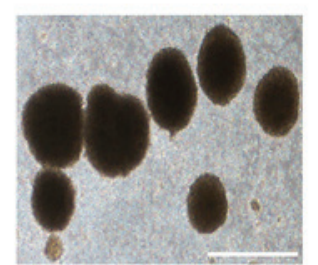

C

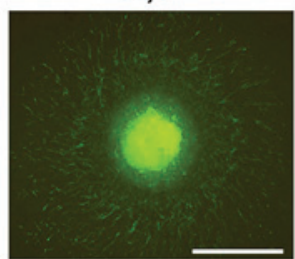

E

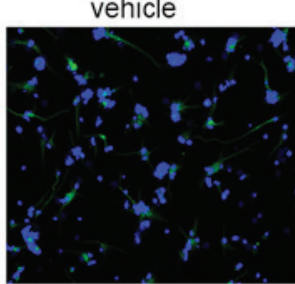

VEGF165

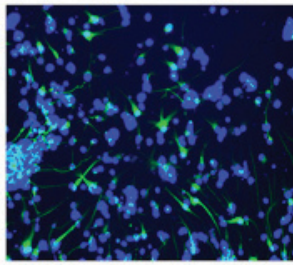

H

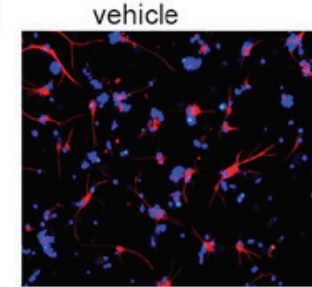

VEGF165

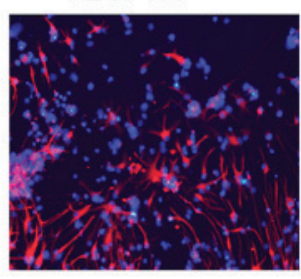

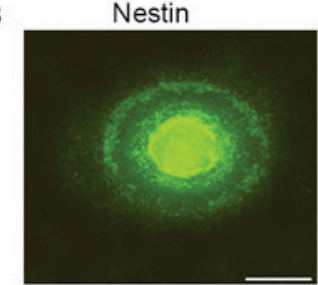

GFAP

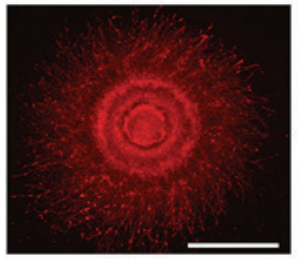

ephrinA1-Fc

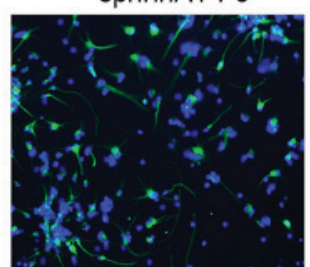

ephrin-A1+VEGF165

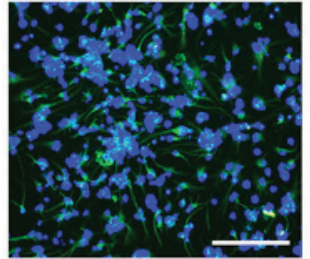

ephrinA1-Fc

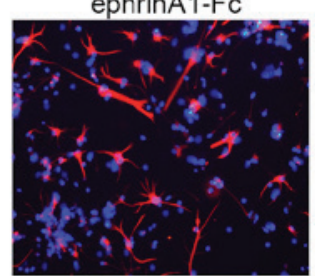

ephrin-A1+VEGF165

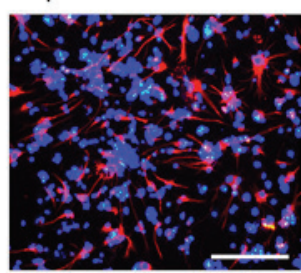

Ki67

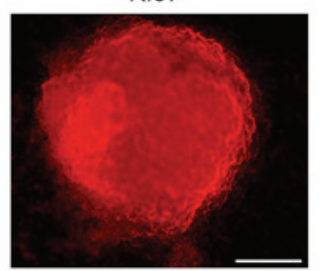

D

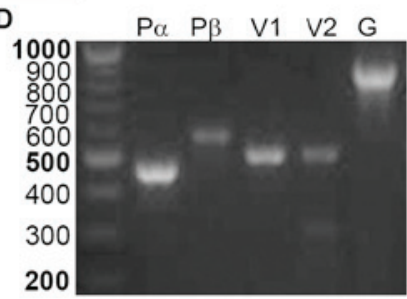

G
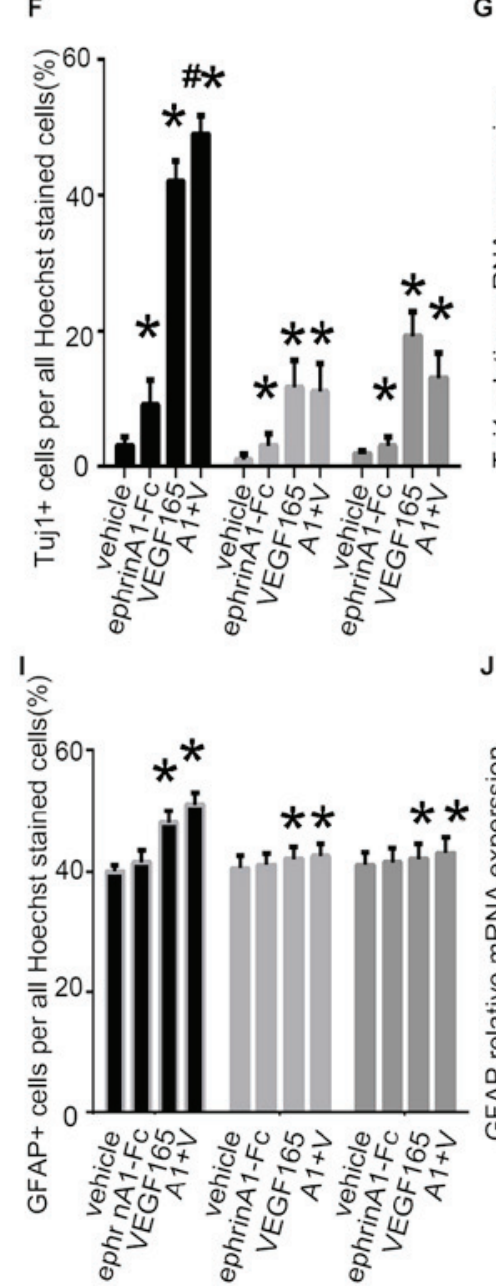

sox2

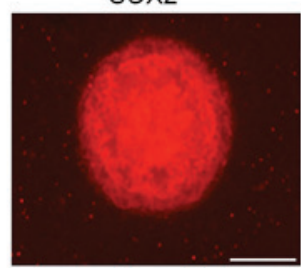

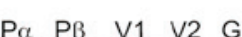
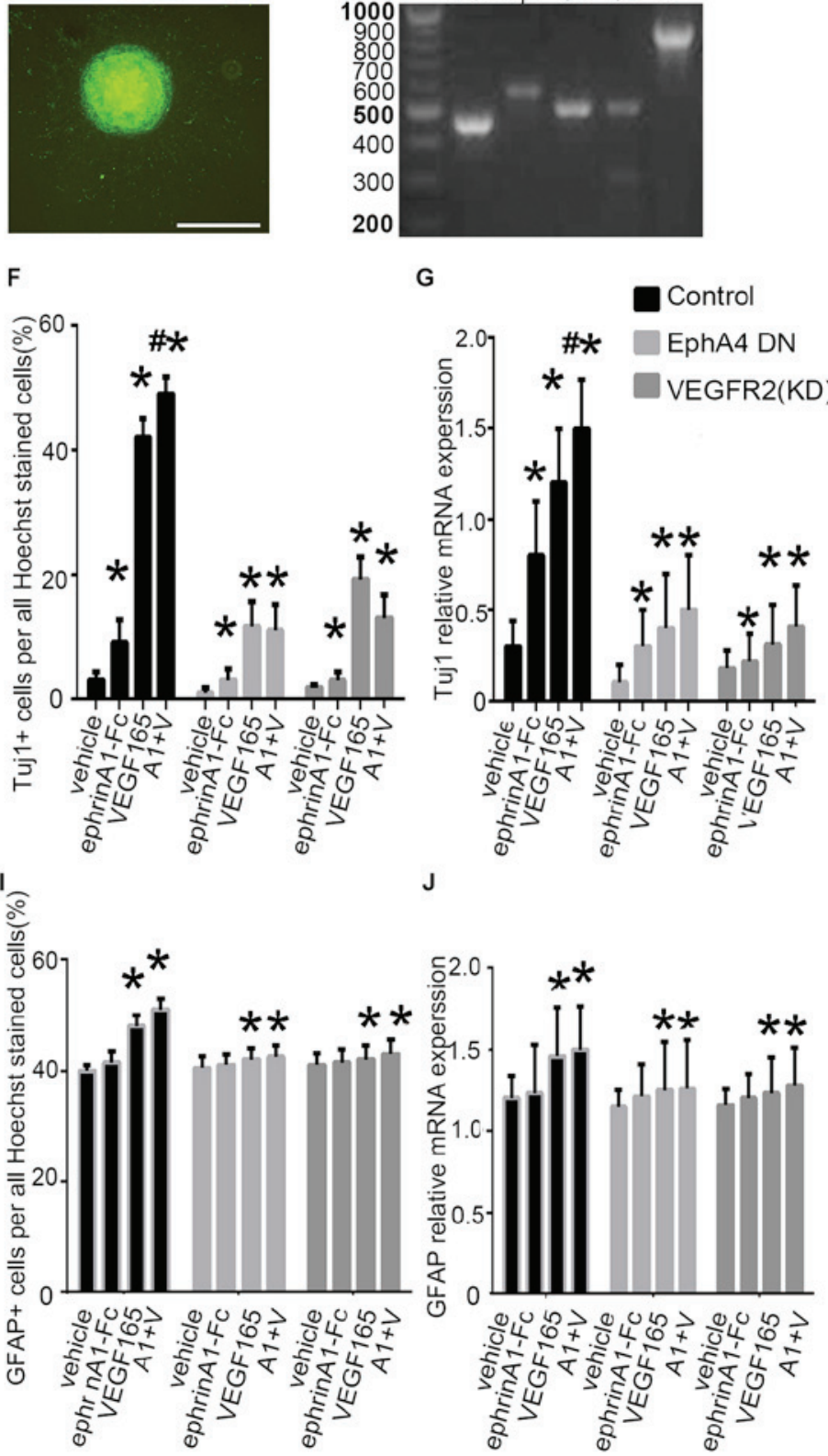

Figure 3. Differentiation of mouse embryonic NSPCs under clustered ephrin-A1-Fc and/or VEGF165 stimulation. (A) Cultured NSPCs grew as neurospheres. (B) Immunofluorescence of the neurospheres show that the cells were nestin ${ }^{+}, \mathrm{Ki}_{67}{ }^{+}$and SOX2 ${ }^{+}$. (C) NSPCs differentiated into neurons (Tuj $1^{+}$), astrocytes $\left(\mathrm{GFAP}^{+}\right)$, and oligodendrocytes $\left(\mathrm{CD} 11 \mathrm{~b}^{+}\right)$. Scale bars, $100 \mu \mathrm{m}$. (D) Expression of VEGFRs and PDGFRs in mouse embryonic NSPCs. Reverse transcription PCR was performed with equal amounts of total RNA isolated from mouse embryonic NSPCs. Fragment lengths are indicated on the left in base pairs. (E) NSPC differentiation was induced under ephrin-A1 stimulation. Tuj $1^{+}$cells in the different groups were stained after culturing for 7 days in normal medium or medium containing clustered ephrin-A1-Fc $(0.5 \mu \mathrm{g} / \mathrm{ml})$ and/or VEGF165 $(20 \mathrm{ng} / \mathrm{ml})$. (F) The proportion of Tuj1 ${ }^{+}$cells per all Hoechst-stained cells in the different treatment groups was analyzed. (G) The mRNA expression levels of Tuj1 in NSPCs cultured in normal medium or medium containing ephrin-A1 and/or VEGF165 was also examined. $(\mathrm{H}) \mathrm{GFAP}^{+}$cells in the different groups were stained after culturing for 7 days in normal medium or medium containing clustered ephrin-A1-Fc $(0.5 \mu \mathrm{g} / \mathrm{ml})$ and/or VEGF165 $(20 \mathrm{ng} / \mathrm{ml})$. (I) The proportion of GFAP cells and (J) mRNA expression levels of GFAP were calculated. NSPCs were transfected with dominant-negative EphA4 mutant or with kinase-negative VEGFR2 mutant prior to stimulation. Data are presented as the mean \pm standard deviation. For $(\mathrm{F})$ and $(\mathrm{I}), \mathrm{n}=3$ in three separate experiments. For $(\mathrm{G})$ and $(\mathrm{J}), \mathrm{n}=5$ in three separate experiments. ${ }^{*} \mathrm{P}<0.05$ vs. the control group; ${ }^{\#} \mathrm{P}<0.05$ vs. VEGF165 treatment alone. DN, dominant negative; Eph, ephrin receptor; fc, fragment crystallizable region; GFAP, glial fibrillary acidic protein; PDGFR, platelet-derived growth factor receptor; NSPC, neural stem and progenitor cell; P $\alpha$, PDGFR $\alpha$; P $\beta$, PDGFR $\beta$; Tuj1, $\beta$-tubulin III; VEGF, vascular endothelial growth factor; VEGFR, VEGF receptor; KD, kinase-dead. A1 + V, ephrin-A1-Fc + VEGF165 stimulation. 
dominant-negative mutant inhibits VEGFR2 phosphorylation at both the basal and the ligand-stimulated phases, these results support the presence of molecular interactions between EphA4 and VEGFR2.

The possibility that EphA4 may crosstalk with VEGFR2 through other growth factor receptors, such as FGFR or PDGFR, cannot be excluded as cross-family interactions have been reported between VEGF/EGFR, PDGF/VEGFR, VEGF-A/PDGFR, PDGFR/FGFR and EphA4/FGFR (25-29). Preliminary immunoprecipitation and immunoblotting experiments also demonstrated that EphA4 and EGFR could interact and transactivate with each other when overexpressed in $293 \mathrm{~T}$ cells (data not shown). For future experiments, the aim is to investigate the downstream signaling pathways associated with the signals mediated by the EphA/FGFR/EGFR/PDGFR/VEGFR complex.

Ephrin-A1 may stimulate dopaminergic neurogenesis and angiogenesis in a 6-hydroxydopamine (6-OHDA) lesioned PD rat model through activating the EphA4 signaling pathway (5). Activating EphA receptors also alters the fate of NSPCs to a neuronal commitment in vitro and in vivo (11). EphA4/ephrin-A signaling serves an important role in establishing the brain vascular system which supports the adult neurogenic niche (30). EphA4 regulates hippocampal neurogenesis via d-serine-regulated N-Methyl-D-aspartic acid receptor signaling in the adult mouse brain (31). As a major angiogenic factor, VEGF promotes neurogenesis in NSPCs in vitro and in the adult brain through the VEGFR2 signaling pathway (13). The proliferative effects of VEGF/VEGFR2 require the ERK and Akt signaling cascades in cultured hippocampal neuronal progenitor cells and in the adult rat hippocampus (32). NSPCs maintain their stem cell proliferative and differentiation ability via self-secreted VEGF interacting with VEGFR2 and VEGF-expressing cells, which in turn provide an enriched environment. This activity may restore functions following brain injuries or in neurodegenerative diseases $(16,33)$. VEGF may trigger spinal cord NSPC proliferation and self-renewal in vitro and the VEGF/VEGFR2/EGFR signaling plays an important role in NSPC activation in vivo (26). Hippocampal administration of VEGF enhances neurogenesis and alleviates the cognitive deficits in immature rats after status epilepticus (34). To the best of the authors' knowledge, the present study is the first demonstration of a potential function of the interaction of Ephs and VEGFRs in NSPC differentiation. Further studies should be designed investigating the efficacy of transplanted NSPCs, treated with combined ephrin-A1 and VEGF165, into several disease animal models in order to identify potential therapies for neurodegenerative diseases such as $\mathrm{AD}$ and PD.

\section{Acknowledgements}

The authors are grateful to Professor Paul Lu from the University of California San Diego for his suggestions in preparing the manuscript.

\section{Funding}

QC is a recipient of a Grant for Doctor from the Shandong Province Scientific Foundation (grant no. ZR2017BH094).

\section{Availability of data and materials}

The datasets used and/or analyzed during the present study are available from the corresponding author on reasonable request.

\section{Authors' contributions}

QFC and FBH conceived and designed the experiments. QFC, JL, TS, CFW and SCW performed the experiments and analyzed the data. QFC and FH wrote the manuscript. All authors approved the final version of this manuscript.

\section{Ethics approval and consent to participate}

All animal experiments were performed in accordance with the guidelines of the Liaocheng People's Hospital (Shandong, China), and were approved by the Ethics Committee of Liaocheng People's Hospital (approval no. LUACC201604).

\section{Patient consent for publication}

Not applicable.

\section{Competing interests}

The authors declare that they have no competing interests.

\section{References}

1. Reynolds BA and Weiss S: Generation of neurons and astrocytes from isolated cells of the adult mammalian central nervous system. Science 255: 1707-1710, 1992.

2. McGrath E, Gao J and Wu P: Proliferation and differentiation of human fetal brain neural stem cells in vitro. J Neurorestoratol 6: 19-27, 2018.

3. Baptista P and Andrade JP: Adult hippocampal neurogenesis: Regulation and possible functional and clinical correlates. Front Neuroanat 12: 44, 2018

4. Liu S and Chen Z: Employing endogenous NSCs to promote recovery of spinal cord injury. Stem Cells Int 2019: 1958631, 2019.

5. Jing X, Miwa H, Sawada T, Nakanishi I, Kondo T, Miyajima M and Sakaguchi K: Ephrin-A1-mediated dopaminergic neurogenesis and angiogenesis in a rat model of Parkinson's disease. PLoS One 7: e32019, 2012.

6. Zhou H, Wei M, Lu L, Chu T, Li X, Fu Z, Liu J, Kang Y, Liu L, Lou Y, et al: Angiopoietin-2 induces the neuronal differentiation of mouse embryonic NSCs via phosphatidylinositol 3 kinase-Akt pathway-mediated phosphorylation of mTOR. Am J Transl Res 11: 1895-1907, 2019.

7. Kullander K and Klein R: Mechanisms and functions of Eph and ephrin signalling. Nat Rev Mol Cell Biol 3: 475-486, 2002.

8. Murai KK and Pasquale EB: Eph receptors, ephrins, and synaptic function. Neuroscientist 10: 304-314, 2004.

9. Wilkinson DG: Multiple roles of EPH receptors and ephrins in neural development. Nat Rev Neurosci 2: 155-164, 2001.

10. Todd KL, Baker KL, Eastman MB, Kolling FW, Trausch AG, Nelson CE and Conover JC: EphA4 regulates neuroblast and astrocyte organization in a neurogenic niche. J Neurosci 37: 3331-3341, 2017.

11. Aoki M, Yamashita T and Tohyama M: EphA receptors direct the differentiation of mammalian neural precursor cells through a mitogen-activated protein kinase-dependent pathway. J Biol Chem 279: 32643-32650, 2004.

12. Huang KF, Hsu WC, Hsiao JK, Chen GS and Wang JY: Collagen-glycosaminoglycan matrix implantation promotes angiogenesis following surgical brain trauma. Biomed Res Int 2014: 672409, 2014.

13. Jin K, Zhu Y, Sun Y, Mao XO, Xie L and Greenberg DA: Vascular endothelial growth factor (VEGF) stimulates neurogenesis in vitro and in vivo. Proc Natl Acad Sci USA 99: 11946-11950, 2002. 
14. Rosenstein JM, Mani N, Khaibullina A and Krum JM: Neurotrophic effects of vascular endothelial growth factor on organotypic cortical explants and primary cortical neurons. J Neurosci 23: 11036-11044, 2003.

15. Hao T and Rockwell P: Signaling through the vascular endothelial growth factor receptor VEGFR-2 protects hippocampal neurons from mitochondrial dysfunction and oxidative stress. Free Radic Biol Med 63: 421-431, 2013.

16. Cao L, Jiao X, Zuzga DS, Liu Y, Fong DM, Young D and During MJ: VEGF links hippocampal activity with neurogenesis, learning and memory. Nat Genet 36: 827-835, 2004.

17. Chen Q, Sawada T, Sakaguchi K and Han F: Direct interaction of receptor tyrosine kinases, EphA4 and PDGFR $\beta$, plays an important role in the proliferation of neural stem cells. J Neurorestoratol 5: 133-141, 2017.

18. Yokote H, Fujita K, Jing X, Sawada T, Liang S, Yao L, Yan X, Zhang Y, Schlessinger J and Sakaguchi K: Trans-activation of EphA4 and FGF receptors mediated by direct interactions between their cytoplasmic domains. Proc Natl Acad Sci USA 102: 18866-18871, 2005.

19. Huang S, Mao J, Ding K, Zhou Y, Zeng X, Yang W, Wang P, Zhao C, Yao J, Xia P and Pei G: Polysaccharides from Ganoderma lucidum promote cognitive function and neural progenitor proliferation in mouse model of Alzheimer's disease. Stem Cell Reports 8: 84-94, 2017.

20. Liu H, Jia D, Fu J, Zhao S, He G, Ling EA, Gao J and Hao A Effects of granulocyte colony-stimulating factor on the proliferation and cell-fate specification of neural stem cells. Neuroscience 164: 1521-1530, 2009.

21. Sawada T, Arai D, Jing X, Furushima K, Chen Q, Kawakami K, Yokote H, Miyajima M and Sakaguchi K: Trans-activation between EphA and FGFR regulates self-renewal and differentiation of mouse embryonic neural stem/progenitor cells via differential activation of FRS2 $\alpha$. PLoS One 10: e0128826, 2015.

22. Vanlandewijck M, Lebouvier T, Andaloussi Mäe M, Nahar K, Hornemann S, Kenkel D, Cunha SI, Lennartsson J, Boss A, Heldin $\mathrm{CH}$, et al: Functional characterization of germline mutations in PDGFB and PDGFRB in primary familial brain calcification. PLoS One 10: e0143407, 2015.

23. Deierborg T, Roybon L, Inacio AR, Pesic $J$ and Brundin P: Brain injury activates microglia that induce neural stem cell proliferation ex vivo and promote differentiation of neurosphere-derived cells into neurons and oligodendrocytes. Neuroscience 171: 1386-1396, 2010.

24. Raff MC, Miller RH and Noble M: A glial progenitor cell that develops in vitro into an astrocyte or an oligodendrocyte depending on culture medium. Nature 303: 390-396, 1983.
25. Mamer SB, Chen S, Weddell JC, Palasz A, Wittenkeller A, Kumar $M$ and Imoukhuede PI: Discovery of high-affinity PDGF-VEGFR interactions: Redefining RTK dynamics. Sci Rep 7: 16439, 2017.

26. Liu SM, Xiao ZF, Li X, Zhao YN, Wu XM, Han J, Chen B, Li JY, Fan CX, Xu B, et al: Vascular endothelial growth factor activates neural stem cells through epidermal growth factor receptor signal after spinal cord injury. CNS Neurosci Ther 25: 375-385, 2019.

27. Chen PY, Simons M and Friesel R: FRS2 via fibroblast growth factor receptor 1 is required for platelet-derived growth factor receptor beta-mediated regulation of vascular smooth muscle marker gene expression. J Biol Chem 284: 15980-15992, 2009.

28. Chen Q, Arai D, Kawakami K, Sawada T, Jing X, Miyajima M, Hirai S, Sakaguchi K and Furushima K: EphA4 regulates the balance between self-renewal and differentiation of radial glial cells and intermediate neuronal precursors in cooperation with FGF signaling. PLoS One 10: e0126942, 2015.

29. Ball SG, Shuttleworth CA and Kielty CM: Vascular endothelial growth factor can signal through platelet-derived growth factor receptors. J Cell Biol 177: 489-500, 2007.

30. Hara Y, Nomura T, Yoshizaki K, Frisén J and Osumi N: Impaired hippocampal neurogenesis and vascular formation in ephrin-A5-deficient mice. Stem Cells 28: 974-983, 2010.

31. Zhao J, Taylor CJ, Newcombe EA, Spanevello MD, O'Keeffe I, Cooper LT, Jhaveri DJ, Boyd AW and Bartlett PF: EphA4 regulates hippocampal neural precursor proliferation in the adult mouse brain by d-Serine modulation of N-Methyl-d-Aspartate receptor signaling. Cereb Cortex 29: 4381-4397, 2019.

32. Fournier NM, Lee B, Banasr M, Elsayed M and Duman RS: Vascular endothelial growth factor regulates adult hippocampal cell proliferation through MEK/ERK- and PI3K/Akt-dependent signaling. Neuropharmacology 63: 642-652, 2012.

33. Kirby ED, Kuwahara AA, Messer RL and Wyss-Coray T: Adult hippocampal neural stem and progenitor cells regulate the neurogenic niche by secreting VEGF. Proc Natl Acad Sci USA 112: 4128-4133, 2015.

34. Han W, Song X, He R, Li T, Cheng L, Xie L, Chen H and Jiang L: VEGF regulates hippocampal neurogenesis and reverses cognitive deficits in immature rats after status epilepticus through the VEGFR2 signaling pathway. Epilepsy Behav 68: 159-167, 2017.

This work is licensed under a Creative Commons Attribution-NonCommercial-NoDerivatives 4.0 International (CC BY-NC-ND 4.0) License. 